\title{
Associations of Health-Related Quality of Life, Fear of Falling and Objective Measures of Physical Function with Bone Health in Postmenopausal Women with Low Bone Mass
}

\author{
Anoohya Gandham ${ }^{1} * \mathbb{C}^{\mathbb{D}}$, Lachlan B. McMillan ${ }^{1}$, Carrie-Anne $\mathrm{Ng}^{1}$, Ludovic Humbert ${ }^{2}$, \\ Maxine P. Bonham ${ }^{3}$, Ayse Zengin ${ }^{1}\left(\mathbb{D}\right.$, Peter R. Ebeling ${ }^{1}$ and David Scott ${ }^{1,4} \mathbb{D}$ \\ 1 Department of Medicine, School of Clinical Sciences at Monash Health, Monash University, Clayton, \\ Victoria 3168, Australia \\ 2 Musculoskeletal Unit, Galgo Medical, 08036 Barcelona, Spain \\ 3 Department of Nutrition, Dietetics and Food, Monash University, Notting Hill, Victoria 3168, Australia \\ 4 Department of Medicine and Australian Institute of Musculoskeletal Science, Melbourne Medical \\ School-Western Campus, The University of Melbourne, St Albans, Victoria 3010, Australia \\ * Correspondence: Anoohya.gandham@monash.edu
}

Received: 7 August 2019; Accepted: 30 August 2019; Published: 2 September 2019

\begin{abstract}
Health-related quality of life (HRQoL) and physical function deteriorate with age and may adversely impact bone health in older adults. We determined associations of objective measures of physical function and HRQoL with bone health in postmenopausal women with low areal bone mineral density (aBMD). Fifty postmenopausal women (64.4 \pm 7.7 years old, mean \pm standard deviation) with low spine, hip or femoral neck aBMD (T- or Z-score $<-1.0$ ) on dual-energy X-ray absorptiometry (DXA) participated. Femoral surface BMD, trabecular, integral and cortical volumetric BMD (vBMD) measurements were obtained using 3D-SHAPER software on DXA. Distal tibial vBMD and microarchitecture were assessed using high-resolution peripheral quantitative computed tomography (HRpQCT). Participants completed self-administered EuroQol-5D (EQ-5D) and modified falls efficacy scale (MFES) questionnaires, and physical function assessments. Stair climb power was positively associated with bone parameters at the hip, femoral neck, and distal tibia (all $p<0.05$ ) in multivariable linear regression. EQ-5D demonstrated no significant associations with bone parameters and MFES was positively associated only with distal tibial cortical vBMD and cortical von Mises stress (both $p<0.05$ ). Objective measures of physical function, particularly muscle power, are more consistently associated with bone parameters compared with self-administered HRQoL questionnaires.
\end{abstract}

Keywords: osteoporosis; postmenopausal women; physical function; bone; health-related quality of life

\section{Introduction}

Osteoporosis is an age-associated disease largely affecting postmenopausal women due to a steep decline in estrogen levels following menopause [1,2]. Osteoporosis is defined as low bone mineral density (BMD) and bone microarchitecture increasing bone fragility and the risk of fracture [3,4]. The current clinical gold standard for diagnosing osteoporosis is a dual-energy $X$-ray absorptiometry (DXA) scan which measures areal BMD (aBMD) $[1,5]$. Although DXA has many advantages, there are a number of limitations, such as space restrictions in a clinical setting and equipment expenses. Hence, the development of cost-effective but reliable methods for identifying individuals at increased risk for poor bone health is important in reducing the burden of osteoporosis. 
Age-related declines in physical function, particularly in postmenopausal women, are sequentially associated with loss of bone and bone mass [6]. Declining physical activity levels with age contribute to bone fragility and an increasing fracture risk in older adults [6,7]. Individuals with better physical function are more capable of engaging in physical activity, which has a protective effect on bone health through mechanical loading [8]. Physical performance assessments can easily be incorporated into a clinical environment given they are generally feasible and have been recommended for risk assessment and diagnosis for a range of conditions, including sarcopenia $[8,9]$. It is possible that they may also have utility for prediction of bone loss in older adults and may be useful for clinicians to identify individuals at risk of poor bone health $[9,10]$.

Health-related quality of life (HRQoL) comprises aspects of physical, emotional and mentalwellbeing which are known to decrease with age [11]. HRQoL instruments are useful and inexpensive assessment tools for overall health status [11,12]. While osteoporosis negatively affects HRQoL in older adults, it is also possible that declines in HRQoL contribute to reductions in bone health, in a bidirectional manner. Fear of falling may also be associated with HRQoL and increases with age resulting in a lack of confidence in performing daily tasks, particularly in older women [12]. Both poor HRQoL and fear of falling may, therefore, be associated with bone health in older adults and could be clinically relevant self-reported measures to identify individuals at increased risk of osteoporosis [13].

The aim of the present study was to determine the associations between self-reported HRQoL, fear of falling, and objective measures of physical function with BMD and bone microarchitecture in postmenopausal women with low aBMD.

\section{Methods}

\subsection{Study Design and Participants}

This analysis utilized baseline data from an exercise intervention conducted at the Monash Health Translation Precinct in Melbourne, Australia. Fifty community-dwelling postmenopausal women residing in Melbourne, Australia were recruited for this study. Recruitment of participants was achieved through advertisements in the local community, hospitals, businesses, and clubs. Inclusion criteria included: T- or Z-score at spine, hip or femoral neck $<-1.0$, body mass index (BMI) $<30 \mathrm{~kg} / \mathrm{m}^{2}$, $<150$ min of self-reported moderate to vigorous physical activity per week, and English-speaking. The study (ANZCTR number: ACTRN12618000101280) was approved by Monash Health Human Research Ethics Committee (HREC/16/MonH/364) and all participants provided written informed consent prior to completing any study assessments.

\subsection{Questionnaires}

At baseline, participants completed self-administered questionnaires including questions on demographics, medication use, disease history, smoking status, and physical activity. HRQoL was measured using the EuroQol-5D (EQ-5D) [12]. The EQ-5D consists of five HRQoL dimensions: Mobility, self-care, usual activities, pain/discomfort, and anxiety/depression. Participants selected one of three responses for each of the dimensions based on which an EQ-5D score was derived to measure the HRQoL status. Scores of the EQ-5D questionnaire range from 1 to 3 , where 1 indicates no problems, 2 indicates some problems, and 3 indicates extreme problems/unable to perform that activity from which a total EQ-5D score (range: 5-15, higher scores indicate poorer HRQoL) has been derived. The modified falls efficacy scale (MFES) was used to assess fear of falls while completing certain tasks $[14,15]$. MFES consists of 14 questions related to a range of daily activities to determine confidence undertaking certain tasks (for example, get in/out of chair) [14]. Participants rated their level of confidence for each of the activities on a scale from 0 to 10, with 0 meaning not confident/not sure and 10 being completely confident/completely sure. The possible range of total scores in the MFES questionnaire was 0 to 140 (with higher scores indicating better confidence) [16]. 


\subsection{Physical Function}

Objective assessments of physical function were performed, including the stair climb power test (SCPT) and the short physical performance battery (SPPB). The SCPT was performed to evaluate leg muscle power and mobility performance [17]. Participants began the test from the base of a 10-stair flight of stairs and were asked to climb the stairs as fast as possible, in a safe manner. The time taken by the participants to ascend the flight of stairs was recorded in seconds using a stopwatch. We then calculated stair climb power using the following formula: power $=$ force $X$ velocity [17]. Force was calculated as the product of participant body mass $(\mathrm{kg})$ and acceleration due to gravity $\left(9.8 \mathrm{~m} / \mathrm{s}^{2}\right)$. Velocity was calculated from the vertical height of stairs $(1.75 \mathrm{~m})$ divided by stair climb time $(\mathrm{s})$. The SPPB test consists of gait speed, chair stand, and standing balance assessments and provides a composite SPPB score $[18,19]$. For the chair stand test, participants were required to stand up straight from a seated position five times as quickly as possible and the time taken was recorded. To complete the gait speed test, participants were required to walk $2.44 \mathrm{~m}$ at a comfortable and normal pace. Gait speed was calculated by distance (walking course $2.44 \mathrm{~m}$ ) and time taken to complete the test (s). For the standing balance tests, the participants were required to hold two positions: Semi-tandem and full-tandem, for $10 \mathrm{~s}$. The semi-tandem stand involves placing the preferred foot in front of the other with the side of the heel from the front foot touching the first toe of the other foot. The full-tandem stand is performed by placing the preferred foot in front of the other and the back of the heel on the front foot touching the toes of the back foot. A total score out of 12 (higher scores indicate better physical function) was calculated based on the performance of the three tests: Chair stand, gait speed, and standing balance assessments [18].

\subsection{Anthropometric, Bone and Blood Biochemistry Parameters}

Participants attended the clinic appointment following an overnight fast of $\geq 10 \mathrm{~h}$. A $20 \mathrm{~mL}$ blood sample was collected by a qualified phlebotomist, and serum samples were analyzed for 25-hydroxyvitamin D (25(OH)D) using the DiaSorin Liaison (DiaSorin Inc, Stillwater, MN, USA) 25(OH)D assay, a direct competitive chemiluminescent immunoassay.

Height $(\mathrm{m})$ and weight $(\mathrm{kg})$ were measured with footwear, headwear, and heavy items of clothing removed. Height was measured using a portable stadiometer (Seca 213 wall-mounted stadiometer, Seca, Hamburg, Germany), weight was measured using an electronic scale (Seca 804, Germany) and body mass index (BMI; $\mathrm{kg} / \mathrm{m}^{2}$ ) was calculated.

Each participant had a DXA scan (Hologic Discovery A, Hologic, Bedford, MA, USA), where aBMD and bone mineral content (BMC) were assessed at the total hip, femoral neck, and lumbar spine using Hologic Apex software version 5.6.0.2. Osteoporosis was classified as T-score $\leq-2.5$ and osteopenia as T-score between -1 and -2.5 , in line with guidelines from World Health Organization [20]. Lumbar spine trabecular bone score (TBS) was assessed using TBS iNsight software version 3.0.2 (Medimaps, Geneva, Switzerland). Using an algorithm, the 3D-SHAPER software (Galgo Medical, Barcelona, Spain) was applied to two-dimensional DXA scans of the proximal femur to model the femoral shape and BMD distribution in three dimensions, and then derived estimates for trabecular, cortical, and integral volumetric BMD (vBMD) and surface BMD, a measure of fracture resistance [21].

High-resolution peripheral quantitative computed tomography (HRpQCT) scans (XtremeCT II, ScanCo, Switzerland) were performed to determine the integral bone quality and strength at the distal tibia [22,23]. Briefly, the non-dominant leg was placed in a cast and stabilized to prevent movement and subsequent artifacts. A scout scan was used to place the reference line at the tibial endplate. A subsequent scan of one hundred and sixty-eight parallel computed tomography slices, encompassing a $9.02 \mathrm{~mm}$ region of the distal tibia was performed with an isotropic resolution of $<55 \mu \mathrm{m}$. HRpQCT scans were then analyzed using the manufacturer's standard protocol, using software version 6.1 . Trabecular and cortical bone parameters derived from HRpQCT included: Trabecular cross-sectional area $\left(\mathrm{mm}^{2}\right)$, trabecular thickness $(\mathrm{mm})$ trabecular separation $(\mathrm{mm}$, the mean space between the trabeculae), cortical cross-sectional area (mm), cortical vBMD, cortical porosity (\%, the segmented 
pore volume) and cortical periosteal perimeter ( $\mathrm{mm})$. [22,23]. Micro-finite element analysis was also performed to assess the effects of a simulated mechanical load to estimate trabecular and cortical von Mises stress (MPa), which is used for fracture prediction with lower values indicating greater fracture resistance, as well as estimated bone failure load $(\mathrm{N})$ and bone stiffness $\left(\mathrm{N} / \mathrm{mm}^{2}\right)[24,25]$.

\subsection{Accelerometer-Determined Physical Activity}

Each participant was provided with an Actigraph GT3X-BT accelerometer (Actigraph, Pensacola, CA, USA) and instructed to wear the accelerometer at the side of the hip for seven consecutive days except during showering, water activities, and sleeping. A diary was also provided, to record the time of the day that the device was worn and removed, time spent outdoors, physical activity performed and any issues that affected their physical activity estimates (for example, health issues resulting in low physical activity). The accelerometer data was recorded at a rate of $50 \mathrm{~Hz}$ and transferred into ActiLife v6.13.3 software for analysis. Accelerometer activity was transformed into counts per minute (CPMs) with an activity threshold of 100 CPMs. The ActiLife software analyzed data in epoch lengths of 60 seconds. Physical activity was categorized based on the following Freedson adult cut points of 1 to 99 CPMs (sedentary), 100 to 1951 CPMs (light) and >1952 CPMs (moderate to vigorous physical activity, MVPA) [26]. MVPA was combined into a single variable due to the low amount of vigorous physical activity recorded in these postmenopausal women [27].

\subsection{Statistical Analysis}

All statistical analyses were performed using SPSS software version 25 (IBM, Chicago, IL, USA). Data related to HRQoL and physical function were non-normally distributed, and so Spearman's correlations were performed to determine associations for self-reported HRQoL (EQ-5D and MFES) and objective measures of physical function (SCPT, SPPB and its components, with the exception of standing balance) with bone parameters. Multivariable linear regression analysis was performed to further investigate these associations after adjusting for confounders including age, MVPA, BMI, and $25(\mathrm{OH}) \mathrm{D}$. For all analyses, $p$-values $<0.05$ were considered statistically significant.

\section{Results}

In total, 50 postmenopausal women were recruited with an age range of 49-82 years (Table 1). Mean BMI was in the normal range with no current smokers in this population and the most common self-reported comorbidities were hypertension and hypercholesterolemia. The majority $(88 \%)$ of participants had sufficient $25(\mathrm{OH})$ D levels $(\geq 50 \mathrm{nmol} / \mathrm{L})$. Twenty-two $(44 \%)$ participants had osteoporosis and twenty-seven (54\%) had osteopenia, based on DXA T-scores. One 49-year-old participant had total hip and lumbar spine Z-scores of -1.5 and -0.9 , respectively. No participants indicated extreme problems or an inability to perform activity in any of the five domains of the EQ-5D questionnaire. Most participants (62\%) had the maximum MFES score of 140, indicating general good health based on the self-reported HRQoL and fear or falling questionnaires. Participants spent almost three-quarters of their accelerometer wear time in sedentary behavior, and only approximately $5 \%$ of their time performing moderate to vigorous intensity activity. Twenty-nine (56\%) participants had an SPPB score of 12/12 and participants had a range of 176-385 $\mathrm{W}$ for the SCPT.

Figure 1 presents the number of participants with either no problem or some problems for each of the five dimensions of EQ-5D, none reported extreme problems. For the self-care domain, all participants reported no problems. The pain/discomfort domain had the highest proportion of participants selecting some problems (36\%). 
Table 1. Participant descriptive characteristics $(n=50)$.

\begin{tabular}{|c|c|}
\hline & Mean \pm Standard Deviation or Percentage \\
\hline Age (years) & $64.4 \pm 7.7$ \\
\hline Weight (kg) & $61.5 \pm 8.5$ \\
\hline Height (cm) & $161.3 \pm 6.5$ \\
\hline BMI $\left(\mathrm{kg} / \mathrm{m}^{2}\right)$ & $23.6 \pm 3.0$ \\
\hline $25(\mathrm{OH}) \mathrm{D}(\mathrm{nmol} / \mathrm{L})$ & $74.6 \pm 25.1$ \\
\hline \multicolumn{2}{|l|}{ aBMD } \\
\hline Total hip $\left(\mathrm{g} / \mathrm{cm}^{2}\right)$ & $0.765 \pm 0.081$ \\
\hline Femoral neck $\left(\mathrm{g} / \mathrm{cm}^{2}\right)$ & $0.636 \pm 0.070$ \\
\hline Lumbar spine $\left(\mathrm{g} / \mathrm{cm}^{2}\right)$ & $0.809 \pm 0.112$ \\
\hline \multicolumn{2}{|l|}{ BMC } \\
\hline Total hip (g) & $26.582 \pm 3.708$ \\
\hline Femoral neck (g) & $3.207 \pm 0.388$ \\
\hline Lumbar spine (g) & $43.585 \pm 10.916$ \\
\hline \multicolumn{2}{|l|}{ Smoking status } \\
\hline Current (\%) & 0 \\
\hline Former $(\%)$ & 22 \\
\hline Never $(\%)$ & 78 \\
\hline \multicolumn{2}{|l|}{ HRQoL and fear of falling } \\
\hline EQ-5D score (out of 15) & $5(5.0,6.3) \#$ \\
\hline MFES score (out of 140) & $140(134.8,140) \#$ \\
\hline \multicolumn{2}{|l|}{ Physical activity } \\
\hline Sedentary time physical activity (\%) & $73.3 \pm 6.0$ \\
\hline Light-intensity physical activity (\%) & $21.7 \pm 4.7$ \\
\hline Moderate-intensity physical activity (\%) & $4.8 \pm 2.4$ \\
\hline Vigorous-intensity physical activity (\%) & $0.2 \pm 0.4$ \\
\hline Very vigorous-intensity physical activity (\%) & $0.004 \pm 0.01$ \\
\hline \multicolumn{2}{|l|}{ Objective measures of physical function } \\
\hline SPPB (out of 12) & $12(11,12) \#$ \\
\hline $\mathrm{SCPT}(\mathrm{W})$ & $277.2(229.1,335.5)$ \\
\hline \multicolumn{2}{|l|}{ Self-reported comorbidities } \\
\hline Hypertension (\%) & 22 \\
\hline Hypercholesterolemia (\%) & 28 \\
\hline Thrombosis $(\%)$ & 2 \\
\hline T2DM (\%) & 4 \\
\hline Asthma (\%) & 14 \\
\hline Bronchitis/emphysema (\%) & 2 \\
\hline Any cancer $(\%)$ & 6 \\
\hline $\begin{array}{l}\text { All data are expressed as mean } \pm \text { SD or frequency }(\%) \text { unl } \\
\text { body mass index; } 25-O H D, 25 \text {-hydroxyvitamin D; aBM } \\
\text { HRQoL, Health-related quality of life; EQ-5D, EuroQo } \\
\text { range; SPPB, short physical performance battery; SCPT }\end{array}$ & $\begin{array}{l}\text { nerwise specified. \# median (IQR). Abbreviations: B } \\
\text { eal bone mineral density; BMC, bone mineral cont } \\
\text { MFES, modified falls efficacy scale; IQR, interquar } \\
\text { climb power test; T2DM, type } 2 \text { diabetes mellitus. }\end{array}$ \\
\hline
\end{tabular}

Table 2 reports Spearman's correlation coefficients for the self-reported EQ-5D and its domains, and MFES, with bone parameters. The EQ-5D domain of self-care was not included in this analysis as all participants selected the same response (score of 1). EQ-5D total score was not correlated with any of the bone parameters but the EQ-5D mobility domain was significantly negatively correlated with cortical $\mathrm{vBMD}$ at the tibia $(p=0.027)$. The pain/discomfort domain was significantly negatively correlated with trabecular vBMD $(p=0.019)$ and integral vBMD at the total hip $(p=0.045)$. MFES was significantly negatively correlated with hip BMC only $(p=0.024)$. 


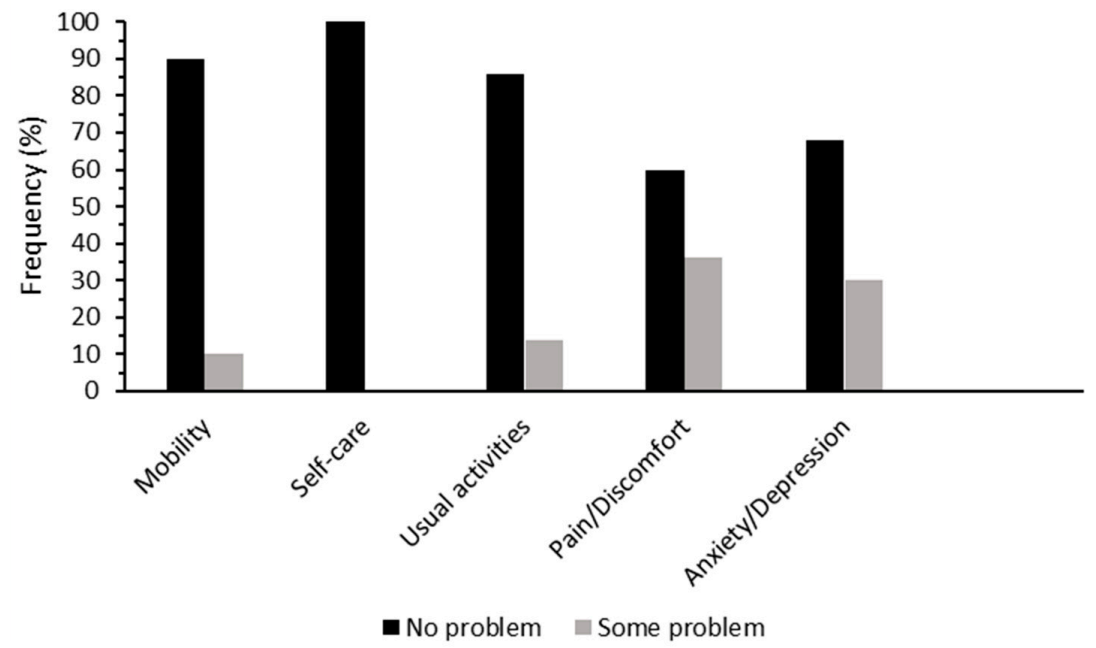

Figure 1. Percentage of postmenopausal women who had no problem (black) and some problems (grey) for the five dimensions of EuroQol-5D (EQ-5D).

Table 2. Spearman's correlations between self-reported measurements and bone parameters.

\begin{tabular}{|c|c|c|c|c|c|c|}
\hline & EQ-5D & Mobility & Usual & Pain & Anxiety & MFES \\
\hline \multicolumn{7}{|l|}{ DXA bone parameters } \\
\hline Hip BMC (g) & 0.264 & 0.132 & 0.222 & 0.158 & 0.269 & $-0.318^{*}$ \\
\hline Hip aBMD $\left(\mathrm{g} / \mathrm{cm}^{2}\right)$ & 0.002 & -0.021 & 0.066 & -0.109 & 0.031 & -0.196 \\
\hline Hip T-score & -0.008 & -0.036 & 0.060 & -0.103 & 0.036 & -0.173 \\
\hline Spine BMC (g) & -0.056 & -0.081 & -0.034 & -0.096 & -0.044 & 0.041 \\
\hline Spine aBMD $\left(\mathrm{g} / \mathrm{cm}^{2}\right)$ & -0.187 & -0.076 & -0.074 & -0.165 & -0.157 & 0.007 \\
\hline Spine T-score & -0.149 & -0.057 & -0.062 & -0.155 & -0.128 & -0.015 \\
\hline LS TBS & -0.033 & -0.039 & -0.056 & -0.045 & 0.103 & 0.062 \\
\hline FN BMC $(\mathrm{g})$ & 0.064 & 0.049 & 0.026 & 0.000 & 0.056 & 0.015 \\
\hline FN aBMD $\left(\mathrm{g} / \mathrm{cm}^{2}\right)$ & -0.066 & -0.025 & -0.078 & -0.118 & -0.053 & 0.095 \\
\hline FN T-score & -0.050 & -0.017 & -0.062 & -0.126 & -0.023 & 0.070 \\
\hline \multicolumn{7}{|l|}{ DXA 3D bone parameters } \\
\hline Surface BMD $\left(\mathrm{mg} / \mathrm{cm}^{3}\right)$ & 0.084 & -0.043 & 0.037 & -0.045 & 0.036 & -0.157 \\
\hline Trabecular vBMD $\left(\mathrm{mg} / \mathrm{cm}^{3}\right)$ & -0.259 & -0.076 & -0.049 & $-0.342 *$ & -0.149 & -0.022 \\
\hline Integral vBMD $\left(\mathrm{mg} / \mathrm{cm}^{3}\right)$ & -0.194 & -0.086 & -0.016 & -0.294 * & -0.149 & -0.078 \\
\hline Cortical vBMD $\left(\mathrm{mg} / \mathrm{cm}^{3}\right)$ & -0.049 & -0.095 & 0.058 & -0.142 & -0.069 & -0.198 \\
\hline Mean cortical thickness (mm) & 0.149 & -0.010 & 0.033 & 0.013 & 0.119 & -0.087 \\
\hline \multicolumn{7}{|c|}{ HR-pQCT bone parameters at distal tibia } \\
\hline Trabecular area $\left(\mathrm{mm}^{2}\right)$ & 0.080 & 0.173 & 0.054 & 0.099 & -0.072 & -0.163 \\
\hline Trabecular thickness (mm) & 0.061 & 0.113 & 0.112 & -0.081 & 0.045 & 0.014 \\
\hline Trabecular separation (mm) & 0.019 & 0.035 & -0.086 & -0.103 & 0.080 & -0.048 \\
\hline Trabecular VMS (MPa) & -0.187 & -0.043 & -0.091 & -0.245 & -0.031 & 0.111 \\
\hline Cortical Area $\left(\mathrm{mm}^{2}\right)$ & -0.023 & -0.146 & -0.182 & -0.023 & -0.011 & 0.081 \\
\hline Cortical vBMD $\left(\mathrm{mg} / \mathrm{cm}^{3}\right)$ & -0.139 & $-0.312 *$ & -0.104 & -0.073 & -0.011 & 0.133 \\
\hline Cortical periosteal perimeter $(\mathrm{mm})$ & 0.022 & 0.148 & 0.110 & 0.042 & -0.180 & -0.142 \\
\hline Cortical porosity $(\%)$ & -0.013 & 0.092 & -0.092 & -0.020 & -0.005 & 0.044 \\
\hline Cortical VMS (MPa) & -0.263 & -0.248 & -0.198 & -0.261 & -0.041 & 0.187 \\
\hline Estimated bone failure load $(\mathrm{N})$ & 0.081 & 0.157 & 0.095 & 0.099 & -0.024 & -0.169 \\
\hline Bone stiffness $(\mathrm{N} / \mathrm{mm})$ & -0.092 & -0.143 & -0.091 & -0.109 & 0.005 & 0.155 \\
\hline
\end{tabular}

All data are presented as Spearman's correlation coefficient ( $p$-value). Abbreviations: DXA, dual-energy X-ray absorptiometry; HRpQCT, high-resolution peripheral quantitative computed tomography; EQ5D, EuroQol-5D; MFES, modified falls efficacy scale; SPPB, short physical performance battery; BMC, bone mineral content; aBMD, areal bone mineral density; LS, lumbar spine; TBS, trabecular bone score; FN, femoral neck; VMS, von Mises stress. * Correlation is significant at the 0.05 level (2-tailed).

Spearman's correlations are presented in Table 3 for relationships between objective measures of physical function and bone parameters. SCPT was significantly positively correlated with a majority of 
the DXA bone parameters including hip BMC and T-score, spine BMC and aBMD, lumbar spine TBS and femoral neck BMC, aBMD, and T-score, and was also significantly correlated with HRpQCT bone parameters, including trabecular area and cortical periosteal perimeter at the distal tibia. However, SCPT was significantly negatively associated with trabecular thickness, estimated bone failure load, and bone stiffness at the distal tibia. Total SPPB score was significantly positively correlated with both lumbar spine TBS and cortical von Mises stress at the distal tibia. Gait speed was significantly negatively correlated with trabecular thickness at the distal tibia while chair stand time was significantly negatively correlated with cortical area and cortical von Mises stress at the distal tibia.

Table 3. Spearman's correlations between objective measurements and bone parameters.

\begin{tabular}{|c|c|c|c|c|}
\hline & Gait Speed (m/s) & Stair Climb Power (W) & Chair Stand Time (s) & SPPB \\
\hline \multicolumn{5}{|l|}{ DXA bone parameters } \\
\hline Hip BMC (g) & 0.272 & $0.390 * *$ & -0.043 & 0.106 \\
\hline Hip aBMD $\left(\mathrm{g} / \mathrm{cm}^{2}\right)$ & 0.234 & 0.249 & -0.172 & 0.211 \\
\hline Hip T-score & 0.226 & 0.296 * & -0.163 & 0.190 \\
\hline Spine BMC (g) & 0.236 & $0.395 * *$ & -0.191 & 0.026 \\
\hline Spine aBMD $\left(\mathrm{g} / \mathrm{cm}^{2}\right)$ & 0.175 & $0.317 *$ & -0.107 & -0.083 \\
\hline Spine T-score & 0.152 & 0.271 & -0.116 & -0.062 \\
\hline LS TBS & 0.159 & $0.344 *$ & -0.262 & 0.299 * \\
\hline FN BMC (g) & 0.220 & $0.405 * *$ & -0.007 & 0.092 \\
\hline FN aBMD $\left(\mathrm{g} / \mathrm{cm}^{2}\right)$ & 0.181 & $0.328 *$ & -0.082 & 0.002 \\
\hline FN T-score & 0.168 & 0.347 * & -0.089 & 0.032 \\
\hline \multicolumn{5}{|l|}{ DXA 3D bone parameters } \\
\hline Surface BMD $\left(\mathrm{mg} / \mathrm{cm}^{3}\right)$ & 0.204 & 0.197 & -0.064 & 0.124 \\
\hline Trabecular vBMD $\left(\mathrm{mg} / \mathrm{cm}^{3}\right)$ & 0.188 & 0.111 & -0.271 & 0.245 \\
\hline Integral vBMD $\left(\mathrm{mg} / \mathrm{cm}^{3}\right)$ & 0.200 & 0.118 & -0.258 & 0.221 \\
\hline Cortical vBMD $\left(\mathrm{mg} / \mathrm{cm}^{3}\right)$ & 0.199 & 0.068 & -0.136 & 0.132 \\
\hline Mean cortical thickness (mm) & 0.203 & 0.279 & -0.029 & 0.139 \\
\hline \multicolumn{5}{|l|}{ HR-pQCT bone parameters at distal tibia } \\
\hline Trabecular area $\left(\mathrm{mm}^{2}\right)$ & 0.151 & $0.342 *$ & 0.153 & -0.045 \\
\hline Trabecular thickness (mm) & $-0.413 * *$ & $-0.314 *$ & 0.097 & -0.109 \\
\hline Trabecular separation (mm) & -0.196 & -0.220 & 0.058 & 0.119 \\
\hline Trabecular VMS (Mpa) & -0.213 & 0.024 & -0.192 & 0.018 \\
\hline Cortical Area $\left(\mathrm{mm}^{2}\right)$ & 0.063 & 0.090 & $-0.341 *$ & 0.087 \\
\hline Cortical vBMD $\left(\mathrm{mg} / \mathrm{cm}^{3}\right)$ & 0.071 & 0.146 & -0.186 & 0.229 \\
\hline Cortical periosteal perimeter $(\mathrm{mm})$ & 0.091 & $0.329 *$ & 0.116 & -0.069 \\
\hline Cortical porosity $(\%)$ & -0.018 & -0.208 & -0.093 & -0.151 \\
\hline Cortical VMS (MPa) & 0.118 & -0.036 & $-0.298 *$ & $0.331 *$ \\
\hline Estimated bone failure load (N) & -0.066 & $-0.314^{*}$ & 0.217 & -0.106 \\
\hline Bone stiffness $(\mathrm{N} / \mathrm{mm})$ & 0.042 & $-0.314 *$ & -0.217 & 0.079 \\
\hline
\end{tabular}

All data are presented as Spearman's correlation coefficient (p-value). Abbreviations: DXA, dual-energy X-ray absorptiometry; HRpQCT, high-resolution peripheral quantitative computed tomography; SPPB, short physical performance battery; BMC, bone mineral content; aBMD, areal bone mineral density; LS, lumbar spine; TBS, trabecular bone score; FN, femoral neck; VMS, von Mises stress. ${ }^{* *}$ Correlation is significant at the 0.01 level (2-tailed). * Correlation is significant at the 0.05 level (2-tailed).

Table 4 presents coefficients from the multivariable linear regression model examining the associations of self-reported HRQoL and objective measures of physical function with bone parameters. After adjustment for confounders, EQ-5D had no significant associations with any of the bone parameters. However, MFES was significantly positively associated with cortical vBMD and cortical von Mises stress at the distal tibia. SCPT remained significantly positively associated with hip BMC, femoral neck BMC, aBMD and T-score, and trabecular area at the distal tibia and was also significantly negatively associated with trabecular thickness at the distal tibia. SPPB was significantly positively associated with cortical von Mises stress at the distal tibia. No associations were found between chair stand time and bone parameters however, gait speed was significantly negatively associated with trabecular thickness and trabecular von Mises stress at the distal tibia. 
Table 4. Multivariable linear regression analysis examining associations between self-reported, objective measurements, and bone parameters.

\begin{tabular}{|c|c|c|c|c|c|c|}
\hline & EQ-5D Score & MFES Score & Gait Speed $(\mathrm{m} / \mathrm{s})$ & Stair Climb Power (W) & Chair Stand Time (s) & SPPB \\
\hline \multicolumn{7}{|l|}{ DXA bone parameters } \\
\hline Hip BMC (g) & $0.218(-0.072,0.509)$ & $-0.175(-0.467,0.117)$ & $0.266(-0.030,0.562)$ & $0.485(0.175,0.795)$ & $-0.157(-0.467,0.153)$ & $0.016(-0.344,0.376)$ \\
\hline Hip aBMD $\left(\mathrm{g} / \mathrm{cm}^{2}\right)$ & $-0.012(-0.327,0.303)$ & $0.032(-0.282,0.346)$ & $0.265(-0.050,0.580)$ & $0.263(-0.093,0.618)$ & $-0.271(-0.592,0.051)$ & $0.243(-0.130,0.616)$ \\
\hline Hip T-score & $-0.026(-0.339,0.288)$ & $0.038(-0.274,0.351)$ & $0.254(-0.061,0.569)$ & $0.239(-0.112,0.591)$ & $-0.251(-0.568,0.066)$ & $0.212(-0.161,0.586)$ \\
\hline Spine BMC (g) & $-0.106(-0.410,0.198)$ & $0.119(-0.184,0.421)$ & $0.219(-0.089,0.527)$ & $0.288(-0.055,0.630)$ & $-0.162(-0.480,0.156)$ & $-0.107(-0.475,0.261)$ \\
\hline Spine aBMD $\left(\mathrm{g} / \mathrm{cm}^{2}\right)$ & $-0.263(-0.566,0.040)$ & $0.204(-0.102,0.510)$ & $0.145(-0.177,0.466)$ & $0.220(-0.137,0.576)$ & $-0.136(-0.464,0.191)$ & $-0.100(-0.478,0.277)$ \\
\hline Spine T-score & $-0.252(-0.562,0.058)$ & $0.211(-0.101,0.523)$ & $0.134(-0.195,0.463)$ & $0.224(-0.135,0.583)$ & $-0.165(-0.494,0.164)$ & $-0.063(-0.450,0.324)$ \\
\hline LS TBS & $-0.020(-0.290,0.249)$ & $0.098(-0.169,0.364)$ & $-0.047(-0.326,0.231)$ & $0.034(-0.278,0.346)$ & $-0.046(-0.329,0.238)$ & $0.052(-0.274,0.377)$ \\
\hline FN BMC (g) & $-0.018(-0.322,0.286)$ & $0.175(-0.123,0.473)$ & $0.294(-0.006,0.594)$ & $0.458(0.136,0.780)$ & $-0.165(-0.481,0.151)$ & $0.268(-0.090,0.625)$ \\
\hline FN aBMD $\left(\mathrm{g} / \mathrm{cm}^{2}\right)$ & $-0.126(-0.434,0.182)$ & $0.250(-0.050,0.550)$ & $0.198(-0.117,0.513)$ & $0.353(0.010,0.696)$ & $-0.205(-0.526,0.116)$ & $0.292(-0.071,0.656)$ \\
\hline FN T-score & $-0.121(-0.431,0.190)$ & $0.245(-0.057,0.547)$ & $0.188(-0.131,0.506)$ & $0.351(0.011,0.692)$ & $-0.206(-0.525,0.113)$ & $0.306(-0.059,0.671)$ \\
\hline \multicolumn{7}{|l|}{ DXA 3D bone parameters } \\
\hline Surface BMD $\left(\mathrm{mg} / \mathrm{cm}^{3}\right)$ & $-0.007(-0.328,0.313)$ & $0.001(-0.308,0.309)$ & $0.211(-0.102,0.524)$ & $0.191(-0.163,0.544)$ & $-0.122(-0.445,0.202)$ & $0.121(-0.251,0.492)$ \\
\hline Trabecular vBMD $\left(\mathrm{mg} / \mathrm{cm}^{3}\right)$ & $-0.246(-0.561,0.070)$ & $0.148(-0.161,0.457)$ & $0.145(-0.176,0.466)$ & $0.075(-0.287,0.437)$ & $-0.252(-0.573,0.068)$ & $0.315(-0.051,0.680)$ \\
\hline Integral vBMD $\left(\mathrm{mg} / \mathrm{cm}^{3}\right)$ & $-0.200(-0.520,0.121)$ & $0.095(-0.218,0.408)$ & $0.151(-0.171,0.474)$ & $0.054(-0.311,0.419)$ & $-0.235(-0.559,0.089)$ & $0.238(-0.136,0.611)$ \\
\hline Cortical vBMD $\left(\mathrm{mg} / \mathrm{cm}^{3}\right)$ & $-0.086(-0.416,0.243)$ & $-0.053(-0.375,0.268)$ & $0.210(-0.118,0.538)$ & $0.068(-0.301,0.437)$ & $-0.170(-0.502,0.162)$ & $0.072(-0.318,0.463)$ \\
\hline Mean cortical thickness (mm) & $0.077(-0.236,0.389)$ & $0.041(-0.264,0.347)$ & $0.191(-0.121,0.502)$ & $0.243(-0.100,0.585)$ & $-0.069(-0.388,0.249)$ & $0.153(-0.215,0.521)$ \\
\hline \multicolumn{7}{|l|}{ HR-pQCT bone parameters at distal tibia } \\
\hline Trabecular area $\left(\mathrm{mm}^{2}\right)$ & $0.161(-0.150,0.472)$ & $-0.215(-0.521,0.092)$ & $0.180(-0.141,0.501)$ & $0.444(0.106,0.781)$ & $0.155(-0.173,0.484)$ & $-0.044(-0.425,0.336)$ \\
\hline Trabecular thickness $(\mathrm{mm})$ & $0.076(-0.249,0.402)$ & $-0.052(-0.376,0.273)$ & $-0.439(-0.745,-0.132)$ & $-0.387(-0.745,-0.029)$ & $0.089(-0.254,0.431)$ & $0.002(-0.393,0.396)$ \\
\hline Trabecular separation (mm) & $0.311(-0.002,0.624)$ & $-0.052(-0.376,0.273)$ & $-0.115(-0.452,0.222)$ & $-0.286(-0.654,0.082)$ & $0.002(-0.342,0.347)$ & $0.336(-0.044,0.717)$ \\
\hline Trabecular VMS (MPa) & $-0.221(-0.539,0.097)$ & $0.078(-0.245,0.401)$ & $-0.322(-0.643,-0.002)$ & $-0.200(-0.567,0.166)$ & $-0.175(-0.509,0.158)$ & $0.028(-0.366,0.421)$ \\
\hline Cortical Area $\left(\mathrm{mm}^{2}\right)$ & $-0.081(-0.400,0.241)$ & $0.218(-0.097,0.534)$ & $0.043(-0.290,0.375)$ & $0.033(-0.340,0.405)$ & $-0.279(-0.606,0.049)$ & $-0.071(-0.459,0.317)$ \\
\hline Cortical vBMD $\left(\mathrm{mg} / \mathrm{cm}^{3}\right)$ & $-0.219(-0.533,0.094)$ & $0.339(0.037,0.641$ & $0.051(-0.281,0.382)$ & $0.062(-0.308,0.433)$ & $-0.168(-0.501,0.166)$ & $0.111(-0.274,0.497)$ \\
\hline Cortical periosteal perimeter $(\mathrm{mm})$ & $0.112(-0.196,0.419)$ & $-0.212(-0.514,0.090)$ & $0.085(-0.234,0.405)$ & $0.447(0.116,0.777)$ & $0.081(-0.244,0.407)$ & $-0.114(-0.486,0.259)$ \\
\hline Cortical porosity $(\%)$ & $0.162(-0.150,0.475)$ & $-0.192(-0.502,0.118)$ & $-0.164(-0.488,0.160)$ & $-0.257(-0.616,0.101)$ & $-0.109(-0.441,0.223)$ & $-0.208(-0.585,0.169)$ \\
\hline Cortical VMS (MPa) & $-0.237(-0.537,0.063)$ & $0.344(0.055,0.633)$ & $-0.006(-0.327,0.315)$ & $-0.188(-0.537,0.161)$ & $-0.254(-0.566,0.058)$ & $0.411(0.061,0.760)$ \\
\hline Estimated bone failure load $(\mathrm{N})$ & $0.125(-0.174,424)$ & $-0.074(-0.374,0.226)$ & $0.034(-0.279,0.347)$ & $-0.147(-0.490,0.195)$ & $0.212(-0.095,0.519)$ & $0.025(-0.341,0.390)$ \\
\hline Bone stiffness $(\mathrm{N} / \mathrm{mm})$ & $-0.138(-0.438,0.163)$ & $0.067(-0.235,0.369)$ & $-0.063(-0.378,0.251)$ & $0.123(-0.223,0.468)$ & $-0.203(-0.513,0.106)$ & $-0.061(-0.428,0.306)$ \\
\hline
\end{tabular}

All data adjusted for age, MVPA, BMI and vitamin D. Bold values are significant at $p<0.05$. Abbreviations: DXA, dual-energy x-ray absorptiometry; HRpQCT, high-resolution peripheral quantitative computed tomography; EQ5D, EuroQol-5D; MFES, modified falls efficacy scale; SPPB, short physical performance battery; BMC, bone mineral content; aBMD, areal bone mineral density; LS, lumbar spine; TBS, trabecular bone score; FN, femoral neck; VMS, von Mises stress. 


\section{Discussion}

Our cross-sectional study in community-dwelling postmenopausal women with low aBMD demonstrates that an objective measure of muscle power is more consistently associated with bone parameters than other clinically relevant objective measures of physical performance, self-reported HRQoL, and fear of falling. This is the first study to compare associations of self-administered questionnaires and objectively measured physical function with bone health using advanced bone imaging. Our data suggest objective assessments, particularly the SCPT, may be used in screening individuals at risk of poor bone health in clinical settings. Given that physical assessments can be easily incorporated in clinical settings, they may serve as useful screening tools to help address current underdiagnosis of osteoporosis [28].

Total EQ-5D scores had no associations with any bone parameters. In contrast, a prior study of 325 women with mean age of 60 years demonstrated that osteoporotic women have poorer HRQoL as estimated by EQ-5D questionnaires, compared with healthy individuals of a similar age [29]. Additionally, a study including 222 participants with mean age of 79 years reported that those with osteoporosis had poorer HRQoL even compared with those with osteopenia, although the HRQoL in this study was measured using the quality of life questionnaire of the International Osteoporosis Foundation (QUALEFFO-41), which specifically measures osteoporosis-related HRQoL [30]. While we did not compare the women with osteopenia or osteoporosis in this study to a control group with normal aBMD, the lack of association between HRQoL and bone parameters in this population is potentially explained by the overall good general health in the current study where the majority of women had either no or few problems for each of the EQ-5D domains. Nonetheless, longitudinal studies with a larger sample size are required to determine whether poorer HRQoL is predictive of an accelerated decline in bone health in older age. It is possible that an osteoporosis-specific HRQoL instrument may be more sensitive in detecting such a relationship.

Despite the lack of association for total EQ-5D scores, the mobility and pain/discomfort domains of EQ-5D were associated with the bone parameters. A study of 1000 Korean women aged $\geq 60$ years reported that individuals with osteoporosis had significantly higher scores in the mobility, pain/discomfort, and usual activities domains of EQ-5D [31]. The lack of association of usual activities HRQoL with bone health in the present study may relate to the fact that only a few women reported some difficulty with usual activities. It is possible that identification of women with reduced mobility and increased pain/discomfort could be a useful strategy to determine an increased osteoporosis risk.

Our study is the first we are aware of to demonstrate an association between higher MFES scores (indicating lower fear of falling) and better cortical bone health, including distal tibial cortical vBMD and cortical von Mises stress. These associations were significant even after adjusting for confounders, including physical activity, in multivariable linear regression analyses suggesting that other factors associated with fear of falling, perhaps including mobility and pain/discomfort, may influence bone health in women with low aBMD. A study of 140 participants reported that fear of falling is increased in older adults with osteoporosis [32]. However, in the current study, the majority of women with low aBMD had the maximum overall score of 140 , indicating the lowest fear of falling. As demonstrated by the objective measures of physical function, and the fact that these women self-selected to participate in an exercise intervention, they likely represent a sample of the postmenopausal population with a relatively low falls risk. Furthermore, although knowledge of osteoporosis and awareness of a diagnosis increases fear of falls in older women [32], the majority of women in the current study were osteopenic rather than osteoporotic, and therefore, less susceptible to fear of falling. Regardless, the association of fear of falling with cortical bone parameters warrants further investigation.

Physical function is potentially an important predictor of fracture risk, largely due to its relationship with falls [33]. Previous studies have also suggested that improved physical performance is associated with better bone health. A study of 3041 older adults reported that physical performance was moderately associated with hip BMD and we previously reported in a study on over 3300 Swedish older adults that better performance in the timed up-and-go test was associated with better distal 
tibial trabecular vBMD assessed by standard peripheral quantitative computed tomography [34,35]. Surprisingly, chair stand time was not associated with any of the bone parameters in this study, and gait speed was significantly negatively associated with trabecular thickness and trabecular von Mises stress at the distal tibia. A study of 116 postmenopausal women observed that gait speed was positively associated with hip aBMD, but similarly found no associations between chair rise time and aBMD [36]. It is possible that gait speed has differing associations with bone compartments at different anatomical sites, given that the hip primarily comprises cortical rather than trabecular bone. A study of 1061 Japanese postmenopausal women reported that gait speed can be used in combination with lower extremity strength measurements as screening tools for low aBMD in postmenopausal women, but based on the findings of the present study, further research is required to explore its associations with bone microarchitectural parameters and vBMD [9]. Previous research has also demonstrated that physical performance tests can improve the predictive ability of existing fracture prediction tools, but the present study is the first we are aware of to indicate that a simple assessment of muscle power, rather than physical performance per se, is more consistently associated with bone health. Thus, future prospective studies should explore whether the addition of muscle power assessment improves fracture prediction compared with other functional assessments [37].

Stimulation of bone formation through resistance exercise is necessary for the maintenance of bone health due to the insufficient mechanical loading obtained from daily activities in older adults [38]. Furthermore, high-intensity resistance training has been shown to improve aBMD and enhance physical function in older women, and this exercise targeting muscle power may be superior to traditional resistance training for maximizing bone health in older age [39]. We observed that SCPT was positively associated with the distal tibial trabecular area, as well as with femoral neck and total hip aBMD. Our findings, therefore, support the concept that improving muscle power may be beneficial for maintaining bone health in older adults, although, as was observed for gait speed, SCPT was negatively associated with trabecular thickness at the distal tibia. It has been reported that increased mechanical loading through physical activity leads to changes to morphological parameters other than trabecular thickness [40], and trabecular number appears more important than trabecular thickness for maintaining bone strength $[41,42]$. Hence, a negative association may not be a clinically significant finding and it should also be noted that trabecular thickness is a derived measurement from HRpQCT rather than a directly measured bone parameter [23]. As a result, assessments of muscle power in clinical practice appears potentially useful for screening for poor bone health in postmenopausal women.

The results of this study are subject to limitations including its cross-sectional design, which limits conclusions on causality of any observed associations. It is likely that poorer bone health contributes to functional decline, poor HRQoL, and fear of falling in postmenopausal women, but we did not ascertain whether previous falls and fractures influenced these relationships. The study had a relatively small sample size and it is unclear whether these results can be generalized to either men or populations with normal aBMD. Hence, longitudinal studies should be conducted in larger populations to confirm if objective measures of physical function are stronger and better predictors of decline in bone health compared with self-reported HRQoL and fear of falls questionnaires. The strengths of the study include a well-defined sample of women with poor bone health, the use of both objective and self-reported measures of physical function, and advanced imaging of bone including HRpQCT.

In conclusion, a clinical assessment of muscle power was more consistently associated with microarchitectural bone parameters compared with other measures of physical function, and self-administered HRQoL and fear of falling questionnaires, in postmenopausal women with low aBMD. In the absence of access to bone imaging devices such as DXA and HRpQCT, and given their concurrent association with risk of falls, it may be beneficial for clinicians to incorporate measures of muscle power in order to screen for poor bone health in older adults. Furthermore, interventions targeting improvements in muscle power may be effective in improving bone health in postmenopausal women at risk of osteoporosis. 
Author Contributions: Conceptualization, P.R.E. and D.S.; methodology, D.S.; formal analysis, A.G.; investigation, C.-A.N. and L.B.M.; resources, P.R.E. and D.S.; data curation, C.-A.N. and L.B.M.; writing-original draft preparation, A.G.; software, L.H., writing-review and editing, A.G., L.B.M., C.-A.N., A.Z., P.R.E. and D.S.; supervision, D.S., A.Z., M.P.B. and P.R.E.; project administration, C.-A.N., L.B.M., and D.S.; funding acquisition, P.R.E. and D.S.

Funding: This research was funded by Monash University, Faculty of Medicine, Nursing and Health Sciences Strategic Grant (SGS16-0388) awarded to D.S. D.S. is supported by a NHMRC RD Wright Biomedical Career Development Fellowship (GNT1123014).

Conflicts of Interest: A.G., L.B.M., C.-A.N., M.P.B., A.Z., P.R.E. and D.S. declare that they have no conflict of interest. L.H. is a stockholder and employee of Galgo Medical.

\section{References}

1. Ji, M.-X.; Yu, Q. Primary osteoporosis in postmenopausal women. Chronic Dis. Transl. Med. $2015,1,9$. [PubMed]

2. Tella, S.H.; Gallagher, J.C. Prevention and treatment of postmenopausal osteoporosis. J. Steroid Biochem. Mol. Boil. 2014, 142, 155-170. [CrossRef] [PubMed]

3. Lips, P.; van Schoor, N.M. Quality of life in patients with osteoporosis. Osteoporos. Int. 2005, 16, 447-455. [CrossRef] [PubMed]

4. Langdahl, B.L.; Ljunggren, Ö.; Benhamou, C.-L.; Marín, F.; Kapetanos, G.; Kocjan, T.; Lespessailles, E.; Napoli, N.; Nikolic, T.; Petto, H.; et al. Fracture Rate, Quality of Life and Back Pain in Patients with Osteoporosis Treated with Teriparatide: 24-Month Results from the Extended Forsteo Observational Study (ExFOS). Calcif. Tissue Int. 2016, 99, 259-271. [CrossRef] [PubMed]

5. Mueller, D.; Gandjour, A. Cost-Effectiveness of Using Clinical Risk Factors with and without DXA for Osteoporosis Screening in Postmenopausal Women. Value Heal. 2009, 12, 1106-1117. [CrossRef] [PubMed]

6. Demontiero, O.; Vidal, C.; Duque, G. Aging and bone loss: New insights for the clinician. Ther. Adv. Musculoskelet. Dis. 2012, 4, 61-76. [CrossRef]

7. Pereira, C.L.N.; Vogelaere, P.; Baptista, F. Role of physical activity in the prevention of falls and their consequences in the elderly. Eur. Rev. Aging Phys. Act. 2008, 5, 51-58. [CrossRef]

8. Berard, A.; Bravo, G.; Gauthier, P. Meta-analysis of the effectiveness of physical activity for the prevention of bone loss in postmenopausal women. Osteoporos. Int. 1997, 7, 331-337. [CrossRef]

9. Sakazaki, T.; Koike, T.; Yanagimoto, Y.; Oshida, Y. Association between gait speed and bone strength in community-dwelling postmenopausal Japanese women. Environ. Heal. Prev. Med. 2012, 17, 394-400. [CrossRef]

10. Beaudart, C.; Rolland, Y.; Cruz-Jentoft, A.J.; Bauer, J.M.; Sieber, C.; Cooper, C.; Al-Daghri, N.; Araujo de Carvalho, I.; Bautmans, I.; Bernabei, R.; et al. Assessment of Muscle Function and Physical Performance in Daily Clinical Practice: A position paper endorsed by the European Society for Clinical and Economic Aspects of Osteoporosis, Osteoarthritis and Musculoskeletal Diseases (ESCEO). Calcif. Tissue Int. 2019, 105, 1-14. [CrossRef]

11. Xu, X.; Ji, W.; Lv, X.-Q.; Zhu, Y.-C.; Zhao, J.-X.; Miao, L.-Z. Impact of physical activity on health-related quality of life in osteoporotic and osteopenic postmenopausal women: A systematic review. Int. J. Nurs. Sci. 2015, 2, 204-217. [CrossRef]

12. McCaffrey, N.; Kaambwa, B.; Currow, D.C.; Ratcliffe, J. Health-related quality of life measured using the EQ-5D-5L: South Australian population norms. Heal. Qual. Life Outcomes 2016, 14, 133.

13. Salaffi, F.; Cimmino, M.A.; Malavolta, N.; Carotti, M.; Di Matteo, L.; Scendoni, P.; Grassi, W. The burden of prevalent fractures on health-related quality of life in postmenopausal women with osteoporosis: The IMOF study. J. Rheumatol. 2007, 34, 34.

14. Aleksic, J.; Zvekic-Svorcan, J.; Vujasinovic Stupar, N.; Jeremic, I.; Grgurevic, A. Cross-cultural validation of the Modified Falls Efficacy Scale in Serbian community-dwelling women at risk for osteoporotic fracture. Menopause 2018, 25, 444-450. [CrossRef] [PubMed]

15. Hazzaa, N.; Naeem, F.; Eldin, H.G.; El Fattah, L.A. Validation of the Modified Falls Efficacy Scale-International (FES-I) in Egyptian Geriatric Population. Egypt. J. Hosp. Med. 2018, 71, 2294-2303.

16. Pua, Y.-H.; Ong, P.-H.; Clark, R.A.; Matcher, D.B.; Lim, E.C.-W. Falls efficacy, postural balance, and risk for falls in older adults with falls-related emergency department visits: Prospective cohort study. BMC Geriatr. 2017, 17, 291. [CrossRef] [PubMed] 
17. Bean, J.F.; Kiely, D.K.; LaRose, S.; Alian, J.; Frontera, W.R. Is Stair Climb Power a Clinically Relevant Measure of Leg Power Impairments in At-Risk Older Adults? Arch. Phys. Med. Rehabil. 2007, 88, 604-609. [CrossRef]

18. Freiberger, E.; De Vreede, P.; Schoene, D.; Rydwik, E.; Mueller, V.; Frändin, K.; Hopman-Rock, M. Performance-based physical function in older community-dwelling persons: A systematic review of instruments. Age Ageing 2012, 41, 712-721. [CrossRef]

19. Guralnik, J.M.; Ferrucci, L.; Pieper, C.F.; Leveille, S.G.; Markides, K.S.; Ostir, G.V.; Studenski, S.; Berkman, L.F.; Wallace, R.B. Lower Extremity Function and Subsequent Disability: Consistency Across Studies, Predictive Models, and Value of Gait Speed Alone Compared with the Short Physical Performance Battery. J. Gerontol. Ser. A Boil. Sci. Med. Sci. 2000, 55, M221-M231.

20. Kanis, J.A. Assessment of fracture risk and its application to screening for postmenopausal osteoporosis: Synopsis of a WHO report. Osteoporos. Int. 1994, 4, 368-381. [CrossRef]

21. Humbert, L.; Martelli, Y.; Fonolla, R.; Steghofer, M.; Di Gregorio, S.; Malouf, J.; Romera, J.; Barquero, L.M.D.R. 3D-DXA: Assessing the Femoral Shape, the Trabecular Macrostructure and the Cortex in 3D from DXA images. IEEE Trans. Med. Imaging 2017, 36, 27-39. [CrossRef] [PubMed]

22. Bouxsein, M.L.; Boyd, S.K.; A Christiansen, B.; E Guldberg, R.; Jepsen, K.J.; Müller, R. Guidelines for assessment of bone microstructure in rodents using micro-computed tomography. J. Bone Miner. Res. 2010, 25, 1468-1486. [CrossRef] [PubMed]

23. Cheung, A.M.; Adachi, J.D.; Hanley, D.A.; Kendler, D.L.; Davison, K.S.; Josse, R.; Brown, J.P.; Ste-Marie, L.-G.; Kremer, R.; Erlandson, M.C.; et al. High-Resolution Peripheral Quantitative Computed Tomography for the Assessment of Bone Strength and Structure: A Review by the Canadian Bone Strength Working Group. Curr. Osteoporos. Rep. 2013, 11, 136-146. [PubMed]

24. Westbury, L.D.; Shere, C.; Edwards, M.H.; Cooper, C.; Dennison, E.M.; Ward, K.A. Cluster Analysis of Finite Element Analysis and Bone Microarchitectural Parameters Identifies Phenotypes with High Fracture Risk. Calcif. Tissue Int. 2019, 105, 252-262. [CrossRef] [PubMed]

25. Boutroy, S.; Van Rietbergen, B.; Sornay-Rendu, E.; Munoz, F.; Bouxsein, M.L.; Delmas, P.D. Finite element analysis based on in vivo HR-pQCT images of the distal radius is associated with wrist fracture in postmenopausal women. J. Bone Miner. Res. 2008, 23, 392-399. [CrossRef] [PubMed]

26. Freedson, P.S.; Melanson, E.; Sirard, J. Calibration of the Computer Science and Applications, Inc. accelerometer. Med. Sci. Sports Exerc. 1998, 30, 777-781. [CrossRef] [PubMed]

27. Menai, M.; Van Hees, V.T.; Elbaz, A.; Kivimaki, M.; Singh-Manoux, A.; Sabia, S. Accelerometer assessed moderate-to-vigorous physical activity and successful ageing: Results from the Whitehall II study. Sci. Rep. 2017, 7, 45772. [CrossRef]

28. Miller, P.D. Management of severe osteoporosis. Expert Opin. Pharmacother. 2016, 17, 473-488. [CrossRef]

29. Dhillon, V.; Hurst, N.; Hannan, J.; Nuki, G. Association of low general health status, measured prospectively by Euroqol EQ5D, with osteoporosis, independent of a history of prior fracture. Osteoporos. Int. 2005, 16, 483-489. [CrossRef]

30. Jahelka, B.; Dorner, T.E.; Terkula, R.; Quittan, M.; Bröll, H.; Erlacher, L. Health-related quality of life in patients with osteopenia or osteoporosis with and without fractures in a geriatric rehabilitation department. Wien. Med. Wochenschr. 2009, 159, 235-240. [CrossRef]

31. Park, H. The impact of osteoporosis on health-related quality of life in elderly women. Biomed. Res. 2018, 29, 3223-3227. [CrossRef]

32. Halvarsson, A.; Franzen, E.; Stahle, A. Assessing the relative and absolute reliability of the Falls Efficacy Scale-International questionnaire in elderly individuals with increased fall risk and the questionnaire's convergent validity in elderly women with osteoporosis. Osteoporos. Int. 2013, 24, 1853-1858. [CrossRef] [PubMed]

33. Resnick, B.; Nahm, E.S.; Zhu, S.; Brown, C.; An, M.; Park, B.; Brown, J. The Impact of Osteoporosis, Falls, Fear of Falling, and Efficacy Expectations on Exercise Among Community-Dwelling Older Adults. Orthop. Nurs. 2014, 33, 277-286. [CrossRef] [PubMed]

34. Taaffe, D.R.; Simonsick, E.M.; Visser, M.; Volpato, S.; Nevitt, M.C.; Cauley, J.A.; Tylavsky, F.A.; Harris, T.B. Lower extremity physical performance and hip bone mineral density in elderly black and white men and women: Cross-sectional associations in the Health ABC Study. J. Gerontol. Ser. A Boil. Sci. Med. Sci. 2003, 58, M934-M942. [CrossRef] [PubMed] 
35. Scott, D.; Johansson, J.; McMillan, L.B.; Ebeling, P.R.; Nordstrom, P.; Nordstrom, A. Associations of Sarcopenia and Its Components with Bone Structure and Incident Falls in Swedish Older Adults. Calcif. Tissue Int. 2019, 105, 26-36. [CrossRef] [PubMed]

36. Lindsey, C.; Brownbill, R.A.; Bohannon, R.A.; Ilich, J.Z. Association of Physical Performance Measures with Bone Mineral Density in Postmenopausal Women. Arch. Phys. Med. Rehabil. 2005, 86, 1102-1107. [CrossRef]

37. Yu, R.; Leung, J.; Woo, J. Incremental Predictive Value of Sarcopenia for Incident Fracture in an Elderly Chinese Cohort: Results from the Osteoporotic Fractures in Men (MrOs) Study. J. Am. Med. Dir. Assoc. 2014, 15, 551-558. [CrossRef]

38. Hong, A.R.; Kim, S.W. Effects of Resistance Exercise on Bone Health. Endocrinol. Metab. 2018, 33, 435-444. [CrossRef]

39. Watson, S.L.; Weeks, B.K.; Weis, L.J.; Harding, A.T.; Horan, S.A.; Beck, B.R. High-Intensity Resistance and Impact Training Improves Bone Mineral Density and Physical Function in Postmenopausal Women with Osteopenia and Osteoporosis: The LIFTMOR Randomized Controlled Trial. J. Bone Miner. Res. 2018, 33, 211-220. [CrossRef]

40. Chang, G.; Pakin, S.K.; Schweitzer, M.E.; Saha, P.K.; Regatte, R.R. Adaptations in Trabecular Bone Microarchitecture in Olympic Athletes Determined by 7T MRI. J. Magn. Reson. Imaging 2008, 27, 1089-1095. [CrossRef]

41. Nikander, R.; Sievänen, H.; Heinonen, A.; Daly, R.M.; Uusi-Rasi, K.; Kannus, P. Targeted exercise against osteoporosis: A systematic review and meta-analysis for optimising bone strength throughout life. BMC Med. 2010, 8, 47. [CrossRef] [PubMed]

42. Silva, M.; Gibson, L. Modeling the mechanical behavior of vertebral trabecular bone: Effects of age-related changes in microstructure. Bone 1997, 21, 191-199. [CrossRef]

(C) 2019 by the authors. Licensee MDPI, Basel, Switzerland. This article is an open access article distributed under the terms and conditions of the Creative Commons Attribution (CC BY) license (http://creativecommons.org/licenses/by/4.0/). 